(peripheral pulmonary stenosis and posterior embryotoxon). At 3 years old liver biopsy revealed paucity of bile ducts, changes typical to AGS, which definitely confirmed the diagnosis.

When the younger, male, sibling presented with typical phenotype and intrahepatic cholestasis in the neonatal period, AGS was immediately suspected. Of associated anomalies, peripheral pulmonary stenosis, posterior embryotoxon and butterflyvertebrae were all present. Stomatology exam revealed hypodontia in both siblings. Brain MRI conducted at age 3 revealed signs of hepatic encephalopaty with no associated vascular anomalies.

Both siblings had severe cholestatic liver disease in preschool period which had stabilized by school age with conservative treatment for pruritus and malabsorption. Both had severe hypercholesterolemia, in the younger sibling it was extreme with LDL cholesterol levels reaching up to 30,1 $\mathrm{mmol} / \mathrm{L}$ ? The levels of bile acids were also extremely high, reaching a maximum of $634,4 \mathrm{umol} / \mathrm{L}$ in the younger sibling. Therapy included chronic use of UDCA, liposoluble vitamins and MCT with a hypercaloric diet.

The natural evolution of the disease is favorable if liver transplantation is avoided until reaching school age. In both our patients, despite severe liver disease, psychosomatic development and quality of life remained intact.

\section{UNUSUAL INCIDENCE OF CRIGLER-NAJJAR SYNDROME TYPE 1 IN CROATIA}

Ivana Todorič ${ }^{\star}$ Mirna Natalija Aničić, Lana Omerza, Irena Senečić-Čala, Duška TješićDrinković, Jurica Vuković. Department of Pediatrics, University Hospital Center Zagreb

\subsection{6/archdischild-2021-europaediatrics.249}

To present patients with Crigler-Najjar syndrome type 1 (CN1). It is a rare autosomal recessive disorder with an incidence of 1: 1000000 live births, characterised by severe unconjugated hyperbilirubinemia which arises as a consequence of the absence of hepatic bilirubin uridine diphosphate glucuronosyl transferase(UGT1A1) activity.

In the last 30 years we treated seven children with this syndrome at the Department of Pediatrics, University Hospital Center Zagreb.

They were from five families- two pairs of siblings (brother and sister) and three unrelated patients (two boys and a girl). Genetic testing of UGT1A1 gene was performed in six patients (two pairs of siblings and two unrelated boys). Unfortunately, one patient's result was lost.

Three patients had frameshift mutations in exon 1: Patient 1 (c.722_723delAG p.Glu241Glyfs*16), Patients 2 and 3 were siblings and had identical mutation (c.717_718delAG p. Q239fsX256). Two patients (4 and 5, also siblings) had identical nonsense mutation in exon 3 (c.1021C>T p.Arg341*).

Genetic testing, as it was not widely available at the time, was not performed in one girl whose diagnosis was made by the chromatographic analysis of bilirubin glucuronides in the bile.

Four patients underwent a liver transplant from living related donors. In two auxillary procedure was performed (siblings at the age of 7 and 9 years) and in two segmental liver transplant (at the age of 6 and 10 years). Prior to surgery, there was also an unsuccessful attempt of hepatocyte transplantation in one patient.

Three liver transplant procedures were successful, and one patient died in the early post-operative course due to primary graft dysfunction.

Three patients who have not yet undergone liver transplant (a 3-year-old boy and two siblings 1.5-year-old girl and her 6month-old brother) are currently treated with phototherapy. At least 10-14 hours long treatment is necessary to keep their bilirubin at an acceptable level (around $250 \mathrm{umol} / \mathrm{L}$ ). Their psychomotor development is appropriate and they have no neurologic impairment.

Considering the number of births per year in Croatia we noticed a remarkably high incidence of $\mathrm{CN} 1$, more than five times as expected $(5,4: 1000$ 000). We don't have explanation for this finding, at least not by mutations observed. Nevertheless, three of our patients are offspring of two families originating in small Croatian enclave in Kosovo where they were isolated for several centuries. Perhaps there are epigenetic factors we are unaware of that may play a role and contribute to this unexpectedly high incidence.

\section{TO BOLUS OR NOT TO BOLUS: A RECURRING SITUATION ENCOUNTERED IN PAEDIATRIC ED}

A Hamid, L Rehman*, T Bolger, I Krebit. Children's Health Ireland

10.1136/archdischild-2021-europaediatrics. 250

To analyse the adherence to guidelines for assessment and management of dehydration in children presenting with acute gastroenteritis/gastritis in a Pediatric ED.

40 charts were reviewed retrospectively over three months for children who had symptoms of vomiting and/or diarrhea. HSE clinical guidelines for assessment and management of Gastroenteritis were used as a standard2.

Specific emphasis was given to the appropriate prescription of normal saline and dextrose boluses3.

Charts were reviewed again after giving appropriate education sessions.

$100 \%$ documentation was noticed for vital signs and capillary refill time.

While making an assessment of dehydration status, degree of dehydration was documented in $17.5 \%$ of cases, this improved to $86 \%$ with massive correction in individual components in degree of dehydration.

Children who required IV fluid, boluses improved from $21.5 \%$ to $95 \%$ for appropriately prescribed saline and dextrose boluses.

Acute Gastroenteritis is a common childhood illness and its severity is linked to etiology, though rotavirus is the most severe infectious agent Dehydration is a frequent association and its severity must be monitored by established score system 1 .

As proven by this audit, assessment and management of Gastroenteritis, although common, can still prove tricky in the acute emergency setting.

However, through education sessions optimal results were achieved. 